\title{
Clinical translation of amide proton transfer (APT) MRI for ischemic stroke: a systematic review (2003-2020)
}

\author{
Lee Sze Foo ${ }^{1}$, George Harston ${ }^{2}$, Amit Mehndiratta ${ }^{3,4}$, Wun-She Yap ${ }^{1}$, Yan Chai Hum ${ }^{1}$, Khin Wee Lai ${ }^{5}$, \\ Shahizon Azura Mohamed Mukari ${ }^{6}$, Faizah Mohd Zaki ${ }^{6}$, Yee Kai Tee ${ }^{1}$ \\ ${ }^{1}$ Lee Kong Chian Faculty of Engineering and Science, Universiti Tunku Abdul Rahman, Kajang, Malaysia; ${ }^{2}$ Oxford University Hospitals NHSFT, \\ Oxford, UK; ${ }^{3}$ Centre for Biomedical Engineering, Indian Institute of Technology Delhi, New Delhi, India; ${ }^{4}$ Department of Biomedical Engineering, \\ All India Institute of Medical Sciences, New Delhi, India; ${ }^{5}$ Faculty of Engineering, Department of Biomedical Engineering, University of Malaya, \\ Kuala Lumpur, Malaysia; ${ }^{6}$ Department of Radiology, Universiti Kebangsaan Malaysia Medical Center (UKMMC), Kuala Lumpur, Malaysia
}

Correspondence to: Yee Kai Tee. Universiti Tunku Abdul Rahman, Jalan Sungai Long, Bandar Sungai Long, Cheras 43000, Kajang, Selangor, Malaysia. Email: teeyeekai@gmail.com.

\begin{abstract}
Amide proton transfer (APT) magnetic resonance imaging (MRI) is a pH-sensitive imaging technique that can potentially complement existing clinical imaging protocol for the assessment of ischemic stroke. This review aims to summarize the developments in the clinical research of APT imaging of ischemic stroke after 17 years of progress since its first preclinical study in 2003. Three electronic databases: PubMed, Scopus, and Cochrane Library were systematically searched for articles reporting clinical studies on APT imaging of ischemic stroke. Only articles in English published between 2003 to 2020 that involved patients presenting ischemic stroke-like symptoms that underwent APT MRI were included. Of 1,093 articles screened, 14 articles met the inclusion criteria with a total of 282 patients that had been scanned using APT imaging. Generally, the clinical studies agreed APT effect to be hypointense in ischemic tissue compared to healthy tissue, allowing for the detection of ischemic stroke. Other uses of APT imaging have also been investigated in the studies, including penumbra identification, predicting long term clinical outcome, and serving as a biomarker for supportive treatment monitoring. The published results demonstrated the potential of APT imaging in these applications, but further investigations and larger trials are needed for conclusive evidence. Future studies are recommended to report the result of asymmetry analysis at $3.5 \mathrm{ppm}$ along with the findings of the study to reduce this contribution to the heterogeneity of experimental methods observed and to facilitate effective comparison of results between studies and centers. In addition, it is important to focus on the development of fast $3 \mathrm{D}$ imaging for full volumetric ischemic tissue assessment for clinical translation.
\end{abstract}

Keywords: Amide proton transfer (APT); chemical exchange saturation transfer (CEST); magnetic resonance imaging; ischemic stroke

Submitted Dec 06, 2020. Accepted for publication Mar 22, 2021.

doi: 10.21037 /qims-20-1339

View this article at: http://dx.doi.org/10.21037/qims-20-1339

\section{Introduction}

Each year about 15 million people suffer from stroke worldwide according to the World Health Organization (WHO) (1). Among this, approximately 5 million die while another 5 million are permanently disabled. According to the American Stroke Association, the most common type of stroke is ischemic stroke caused by an occlusion of a blood vessel supplying the brain, accounting for $87 \%$ of all strokes $(2,3)$. Without timely reperfusion, ischemia will lead to cerebral infarction due to a prolonged impairment of oxygen and nutrient supply. 
Early intervention and reperfusion have been the mainstay of acute stroke treatment (4). Although there have been many attempts to develop neuroprotection therapies, none have been successfully translated into routine clinical practice (5). The failures are multifactorial, for example, the inability to properly account for patient heterogeneity and a lack of reliable and clinically proven surrogate outcomes (6). In order to better stratify acute ischemic stroke patients for treatment decisions, imaging biomarkers are increasingly being used to better understand the pathophysiology of acute ischemic stroke (7).

The main aim of recanalization therapy is to recover tissue that may be salvaged through timely reperfusion and minimize the final area of infarction. This viable tissue is referred to as the ischemic penumbra (8). The current method of estimating the penumbra includes determining the spatial mismatch between the infarct core estimate and hypoperfused area. The most widely used imaging modality to perform this quantification is through computed tomography (CT) and CT perfusion (CTP) imaging due to its speed and wide availability $(9,10)$. Alternatively, the penumbra has also been estimated using magnetic resonance imaging (MRI), via the mismatch between diffusionweighted imaging (DWI) and perfusion-weighted imaging (PWI) (11).

However, the infarct core and hypoperfused area mismatch can often lead to inaccuracies in the extent of the ischemic penumbra, falsely including the benign oligemia (12) or overestimating the ischemic core (so-called "ghost core") $(13,14)$. According to the pathophysiological evolution of ischemic stroke, tissue acidification happens prior to cerebral infarction (15). Thus, it is often hypothesized that a $\mathrm{pH}$-sensitive imaging technique may be able to act as a more accurate biomarker for identifying the penumbra.

Chemical exchange saturation transfer (CEST) MRI is a novel $\mathrm{pH}$-weighted imaging technique first demonstrated by Balaban et al. in 2000 that enables the indirect detection of low concentration endogenous molecules in a non-invasive manner via chemical exchange with water protons $(16,17)$. To date, the most studied form of CEST MRI involves the detection of exchangeable amide protons in the backbone of mobile proteins and/or peptides, aptly called amide proton transfer (APT) MRI (18-21). Due to the $\mathrm{pH}$-weighting of CEST and thus APT MRI, this imaging technique has the potential to complement the existing imaging modalities in ischemic stroke imaging, particularly, in the definition and investigation of the penumbra.
The first study demonstrating the use of APT MRI in acute ischemic stroke was reported by Zhou et al. in 2003 (22). The study revealed $\mathrm{pH}$-sensitive APT effects in rat global ischemia models and demonstrated that APT signal was reduced in the induced ischemic area, consistent with pathophysiological evolution of ischemic stroke. When ischemia occurs, cerebral blood flow is impaired, leading to lack of metabolic supply, subsequently tissue hypoxia, acidification due to adenosine triphosphate breakdown (23), and if prolonged, cerebral infarction $(21,24)$.

Soon after the first preclinical study by Zhou et al. (22), reports on the characterization of the ischemic penumbra followed (25), demonstrating the potential of APT imaging in delineating the ischemic injury zones: the irreversible ischemic core, the salvageable penumbra, and the benign oligemia. Following this, many studies have been published on the potential use of APT imaging for stroke diagnosis (26-28), disease progression (29), and treatment monitoring (30) throughout the years.

The present review aims to summarize the journey of APT MRI in clinical stroke imaging after 17 years (2003-2020), report the prevalence of APT MRI applied in ischemic stroke patients, the breakthroughs in the literature, limitations in the various reported studies and how the future works can be streamlined to advance the use of APT MRI for stroke diagnosis.

\section{Methods}

This study aimed to identify all published clinical studies documenting the use of APT MRI for the investigation of ischemic stroke in human patients. The studies were compiled according to a pre-specified protocol that outlines our search strategy and inclusion criteria, and quality assessment in accordance to the Preferred Reporting Items for Systematic reviews and Meta-Analyses (PRISMA) guidelines for systematic reviews (31).

\section{Study selection}

To be included in this review, a study must satisfy the following criteria:

(I) Original study that was published in peer-reviewed journals, excluding reviews;

(II) Study must involve human patients that exhibited ischemic stroke-like symptoms; animal only studies are excluded;

(III) Ischemic stroke patients must have undergone 


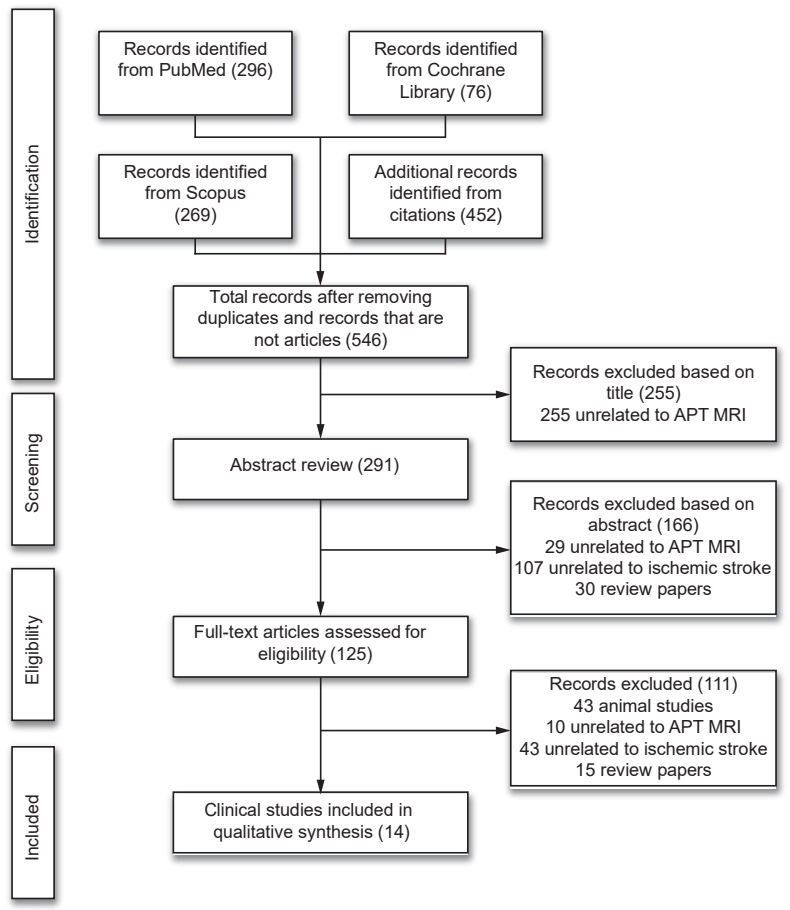

Figure 1 Summary of inclusion/exclusion strategy.

APT MRI;

(IV) Results on APT MRI must be reported;

(V) Articles written in English only.

Three electronic databases-PubMed, Scopus, and Cochrane Library (Cochrane Central Register of Controlled Trials), were searched for clinical studies on APT MRI of patients with ischemic stroke. The built-in advanced search builder in the database websites were used. The search terms used were: ((chemical exchange saturation transfer magnetic resonance imaging) OR (CEST magnetic resonance imaging) OR (CEST MRI) OR (chemical exchange saturation transfer) OR (CEST) OR (amide proton transfer magnetic resonance imaging) OR (APT magnetic resonance imaging) OR (APT MRI) OR (amide proton transfer) OR (APT)) AND ((stroke) OR (ischemic stroke) OR (ischemic) OR (ischemia)). The search was further filtered to show only papers in English and papers published between 1st January 2003 and 31st December 2020. Additionally, Google Scholar was used to check for related articles in the citing literature of the included studies.

After performing the search, articles were reviewed in increasing specificity to remove articles not fulfilling the set criteria via the title, then abstract, and finally the full text. Spreadsheet software was used to organize and assess the included study titles to remove duplicated entries. The abstracts were viewed through word-processing software. An article would be rejected if the title was irrelevant and if the abstract clearly failed to satisfy the set inclusion criteria. After shortlisting the search, the full text of each candidate article was reviewed to determine the final included studies. Any discrepancies in the results of the review were discussed in team meetings.

\section{Stroke classification}

Stroke has been categorized into different stages using several definitions. In this review, the classification was set according to the agreed definitions established in the Stroke Recovery and Rehabilitation Roundtable as reported by Bernhardt et al. (32):

(I) Hyperacute, 0-24 hours from symptom onset;

(II) Acute, 1-7 days;

(III) Early subacute, 7 days-3 months;

(IV) Late subacute, 3-6 months;

(V) Chronic, more than 6 months.

\section{Results}

A total of 1,093 published articles were identified in the search, as shown in Figure 1. After removing duplicates and records that were not journal articles, a total of 546 articles were identified, of which, 255 were excluded based on the title, leaving only 291 articles to screen. Through the abstract screening, only 125 articles were determined to be relevant. Among the 125 articles, only 14 were clinical studies involving human patients with ischemic stroke; the other 111 articles either only involved the use of animal stroke models (43 papers), were unrelated to APT MRI (10 papers), were unrelated to ischemic stroke imaging (43 papers), or were review papers (15 papers).

\section{Clinical Studies from 2003-2020}

Figure 2 summarizes the included clinical studies related to the use of APT imaging in ischemic stroke patients and the timeline in which they were published. The number of published clinical studies showed a gradual increase over the years. In the 14 studies, a total of 282 patients had been scanned with APT imaging after accounting for those judged to be duplicate analyses (using the same patient data but for different purposes); the study by Harston et al. (33) and two studies by Msayib et al. $(34,35)$ used data from the 


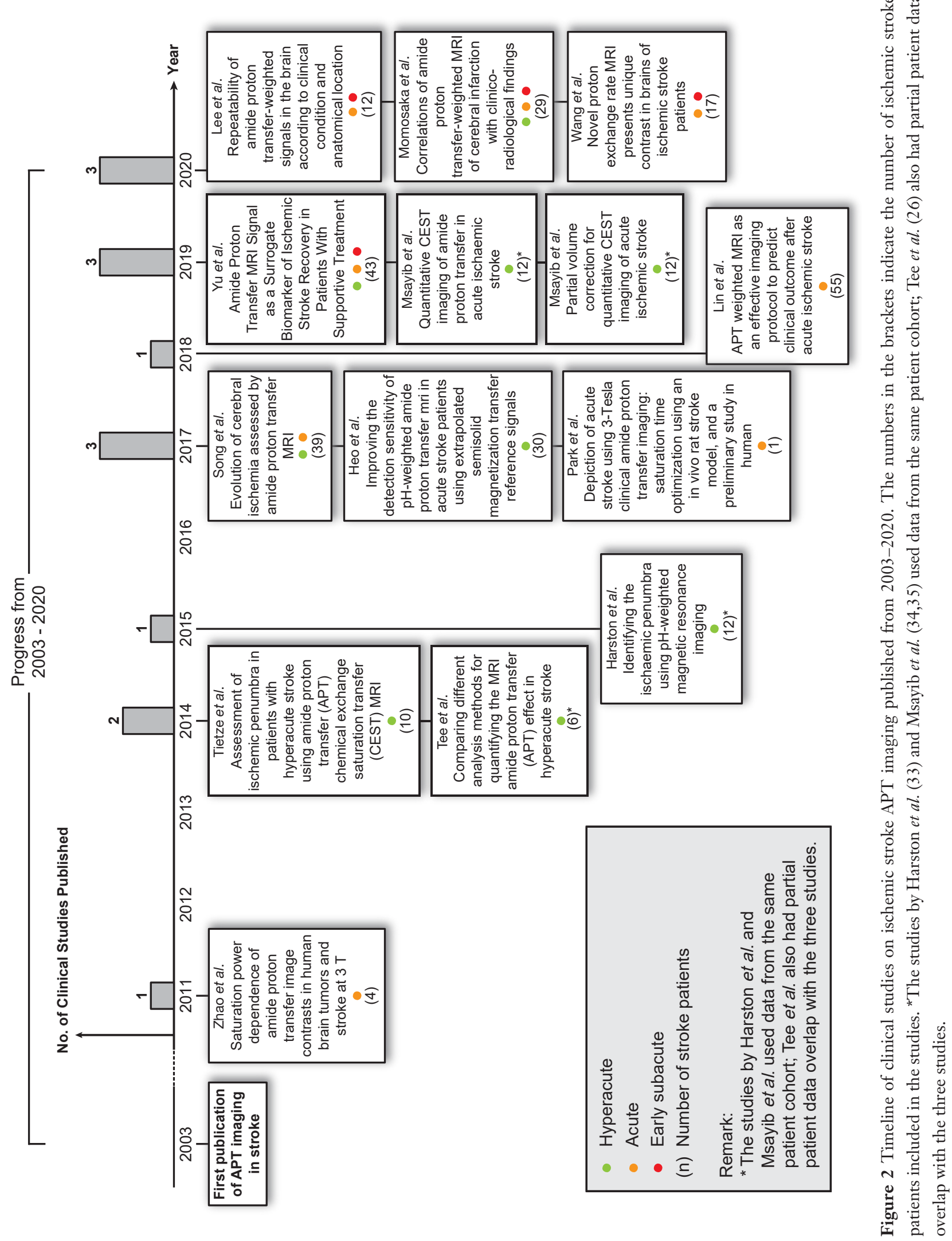


same patient cohort, while the study by Tee et al. (26) also had partial patient data overlap with the three studies.

Six of the studies included only hyperacute stroke patients (26,33-37), three studies involved only acute stroke patients $(29,38,39)$, one study included both hyperacute and acute patients (40), two studies involved acute and early subacute patients $(41,42)$, while two studies involved patients from the hyperacute to early subacute stages $(30,43)$. Further information on these studies such as the treatment received by patients, scanner field strength, radio frequency (RF) saturation scheme, imaging acquisition parameters, image acquisition time, APT effect quantification method, and other MRI sequences acquired, are presented in https://cdn.amegroups.cn/static/public/qims-20-1339-1. pdf $(44,45)$. For further details on the APT quantification methods e.g., the definitions as well as the respective advantages and limitations, please refer to Foo et al. (46).

After the initial preclinical study on APT imaging in 2003, the first clinical study on APT imaging of ischemic stroke patients was published by Zhao et al. in 2011 (38). The study proposed an optimized RF saturation scheme with the aim of establishing a standardized saturation scheme for following clinical studies. In Zhao's study, the APT effect in four acute stroke patients were quantified, revealing APT signal in stroke lesions to appear hypointense compared to normal-appearing white matter (NAWM), consistent with the first animal stroke studies (22), thus demonstrating the feasibility of APT imaging of stroke patients on a clinical field strength scanner.

Later in 2014, Tietze et al. (36) published a clinical study investigating the ability of APT imaging to distinguish the ischemic penumbra from the infarct core and oligemia in hyperacute patients, but yielded inconclusive results. In the same year, Tee et al. (26) performed a study comparing quantitative Bloch McConnell model-based analysis and conventional asymmetry analyses, and found that the model-based analysis was able to produce higher contrastto-noise ratio (CNR) than the asymmetry analyses. Using the model-based method, a relationship between the quantified APT effect and $\mathrm{pH}$ was determined, producing the first quantitative $\mathrm{pH}$ maps of ischemic stroke patients generated via APT MRI.

In 2015, a proof of concept study on identifying the ischemic penumbra was published by Harston et al. (33). Contrary to the study by Tietze et al. (36), the quantified APT effect within the ischemic core was found to be significantly lower than that in the infarct growth, which in turn was lower than in the oligemia regions. This trend of reduction in the three injury zones demonstrated the potential of APT imaging for delineating the ischemic lesion.

Later in 2017, Song et al. (40) conducted a study on assessing the dynamic changes of APT effect during different stages of stroke. The study found APT effect in ischemic tissue to increase with onset time, reducing the contrast between ischemic and non-ischemic tissue as time progressed. In the same year, Heo et al. (37) published a study on improving the quantification of APT effect in ischemic tissue by using extrapolated semisolid magnetization transfer reference (EMR) data analysis, which was found to produce higher contrast between ischemic and non-ischemic tissue compared to asymmetry analysis. In another study, Park et al. (39) sought to optimize the saturation time for a three-dimensional (3D) turbo spin-echo (TSE) acquisition and Sinc-Gaussian saturation scheme. The optimized saturation scheme was then applied on an acute stroke patient as a proof of concept study.

In 2018, Lin et al. (29) published a paper on using APT imaging for assessing stroke severity and for predicting clinical outcome of acute stroke patients. The difference in APT signals within the ischemic and contralateral tissues at presentation was found to have significant inverse correlation with the National Institutes of Health Stroke Scale (NIHSS) and 90-day modified Rankin Scale (mRS) scores. Lesion APT signal was also significantly lower in the groups with poor prognosis compared to those with good prognosis. The study demonstrated the potential of APT imaging to predict patient prognosis and long-term clinical outcome upon imaging 24-48 h from symptom onset.

Then in 2019, Yu et al. (30) proposed the use of APT imaging as a biomarker of ischemic stroke recovery in patients receiving supportive treatment. APT effect was found to have significant correlation with post-treatment time, and the increase in APT effects post-treatment was associated with clinical symptom improvements while the opposite was observed in patients exhibiting aggravated symptoms, highlighting the potential of APT imaging in determining treatment efficacy of ischemic stroke treatment.

In the same year, Msayib et al. (34) published a study comparing APT quantification methods for optimal ischemic stroke imaging. The study found multi-pool Bloch McConnell model-based method to provide the highest CNR between the ischemic core and contralateral tissue among the tested methods, similar to the findings of Tee et al. (26). In a separate study, Msayib et al. (35) also 
investigated the use of a partial volume correction (PVC) model to correct for partial volume effects (PVE). In the low PVE voxels, the PVC model produced a significantly decreased ischemic core signal. However, when analyzing the whole image slice (including low and high PVE voxels), similar results were observed with or without the use of PVC.

Recently in 2020, Lee et al. (41) published a study investigating the repeatability of APT imaging of the brain during different clinical conditions and anatomical locations. With the tested imaging acquisition parameters and APT quantification method in the study, the stroke lesions imaged proved to have excellent repeatability among both intra- and inter-session scans.

Momosaka et al. (43) later published a detailed investigation of the relationship between APT effect with clinico-radiological findings in ischemic stroke patients. It was discovered that APT effect was lower in the poor prognosis group compared to the good counterpart. The signal was also lower in patients with large infarctions, infarctions with low apparent diffusion coefficient (ADC), shorter time after onset, and high NIHSS and mRS scores, in line with the study by Lin et al. (29).

Lastly in the same year, Wang et al. (42) published a study quantifying the average proton exchange rate $\left(k_{\mathrm{ex}}\right)$ of all exchangeable proton species within brain tissue using an omega plot analysis. The quantified $k_{\text {ex }}$ may serve as a potential surrogate imaging biomarker for metabolic changes of stroke and help for monitoring the treatment and evolution of stroke.

\section{APT effect in ischemic lesions}

Figure 3 shows representative APT images of ischemic stroke patients adapted from the included studies $(26,29,30,33,40,43)$. Generally, the clinical studies shared the consensus that APT effect in ischemic tissue (inclusive of the ischemic core, penumbra, and benign oligemia) is smaller compared to normal healthy tissue in early stroke imaging. This produces hypointense APT signals in the ischemic areas (Figure 3), as was reported in all the studies, in agreement with preclinical findings using animal models $(47,48)$.

In the included studies, it was consistently hypothesized that the reduced APT effect is a result of tissue acidosis. Since APT is base-catalyzed and thus $\mathrm{pH}$-dependent, APT effect is reduced within ischemic tissue that underwent tissue acidification as a result of impaired cerebral blood flow. This decrease in intracellular $\mathrm{pH}$ in ischemic tissue was estimated in the quantitative $\mathrm{pH}$ map generated by Tee et al. (26), as shown in Figure $3 A$, where the absolute $\mathrm{pH}$ was quantified through a relationship between quantified APT effect and $\mathrm{pH}$ level.

Six of the included studies involved only hyperacute patients, scanned within 24 hours from onset (26,33-37), whereas the other eight studies involved patients that were scanned outside of this time frame (29,30,38-43). Among which, three studies investigated the progression of the disease with time, namely the studies by Song et al. (40), Yu et al. (30), and Momosaka et al. (43). All three studies found APT signal within the ischemic lesion to gradually increase with increasing time from symptom onset. In addition, Yu et al. further investigated the progression of APT signal pre- and post-supportive treatment (https://cdn.amegroups. cn/static/public/qims-20-1339-1.pdf) and observed that post-treatment, APT signal was found to increase beyond the non-ischemic tissue such that the APT signal in the ischemic lesion became hyperintense when imaged $\geq 8$ days post-treatment.

In the studies by Song et al. (40), Yu et al. (30), and Momosaka et al. (43), the authors attributed the increase in APT effect days after symptom onset to an increase of intracellular $\mathrm{pH}$. However, these deductions may only be partially true since it is difficult to estimate the degree to which intracellular $\mathrm{pH}$ was acting as the main contributing factor to the increased APT effect. As mentioned in the initial paper by Zhou et al., the drop in $\mathrm{pH}$ assumption is potentially valid during the hyperacute stage since water relaxation time and amide proton concentration should have negligible change shortly after the stroke onset (22).

Nevertheless, the changes in water diffusivity or protein properties may influence the APT signal even in the acute stage. Inflammation as well as edema formation are also common occurrences following stroke which can change the magnetic properties of water. Additionally, regular changes in the brain such as protein synthesis and degradation, and the loss of cytoplasm days after the onset might alter the amide proton concentration. All these factors need to be taken into consideration; merely relating the increase in APT signal to $\mathrm{pH}$ changes may not be warranted especially in the later stages of stroke and more works are required to verify the association at all the time points.

\section{Identifying the ischemic penumbra}

Of the 14 clinical studies included, four investigated the 

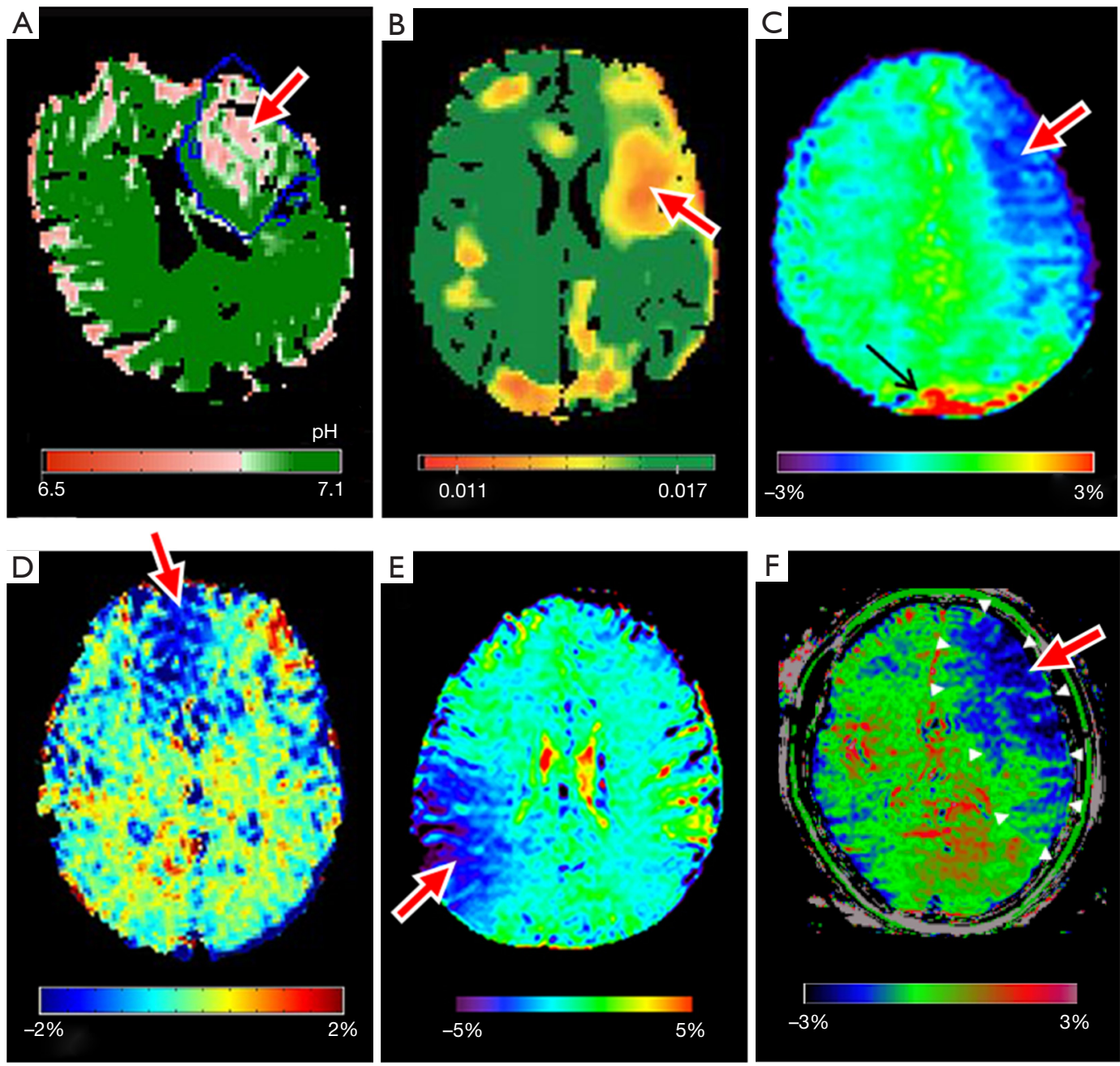

Figure 3 Representative APT images adapted from the clinical studies. All the APT images show hypointensity within the ischemic regions compared to normal brain tissue indicated by the red arrow; black arrow shows the artifacts. (A) quantitative pH map of a hyperacute stroke patient at $1 \mathrm{~h} 43 \mathrm{~m}-5 \mathrm{~h} 46 \mathrm{~m}$ post-onset, adapted from Tee et al. (26); the blue line outlines the infarct core; (B) pH-weighted image of a hyperacute stroke patient scanned at $2 \mathrm{~h} 48 \mathrm{~m}$ after onset, adapted from Harston et al. (33); (C) APT-weighted image of a hyperacute stroke patient at $2 \mathrm{~h}$ from onset, adapted from Song et al. (40); (D) APT-weighted image of an acute stroke patient imaged 24-48 h post symptom onset, adapted from Lin et al. (29); (E) APT-weighted image of an acute stroke patient scanned at 1 day post onset, adapted from Yu et al. (30). (F) APT-weighted image of a hyperacute stroke patient scanned at 15h post onset, adapted from Momosaka et al. (43). All images were reproduced with permission from the publishers.

ability of APT imaging in distinguishing the ischemic penumbra from the core and the oligemia regions, namely the studies by Tietze et al. (36), Harston et al. (33), Heo et al. (37), and Msayib et al. (34). However, there were mixed results reported among the studies. Representative results from two of the studies $(33,34)$ are shown in Figure 4.

Tietze et al. (36) published the first clinical study comparing three regions of interest (ROIs): ischemic core, final infarct growth and at-risk tissue. In their study, inconclusive results were observed as only some patients displayed a trend of reduced APT signal in the ischemic core compared to the at-risk tissue. Upon performing group analysis, although there were significant differences between each of the ROIs with NAWM, there were no significant differences between any of the ischemic ROIs, even after excluding potential outliers (36).

In contrast to the Tietze's group, the study by Harston et al. (33) reported significant differences between the three 

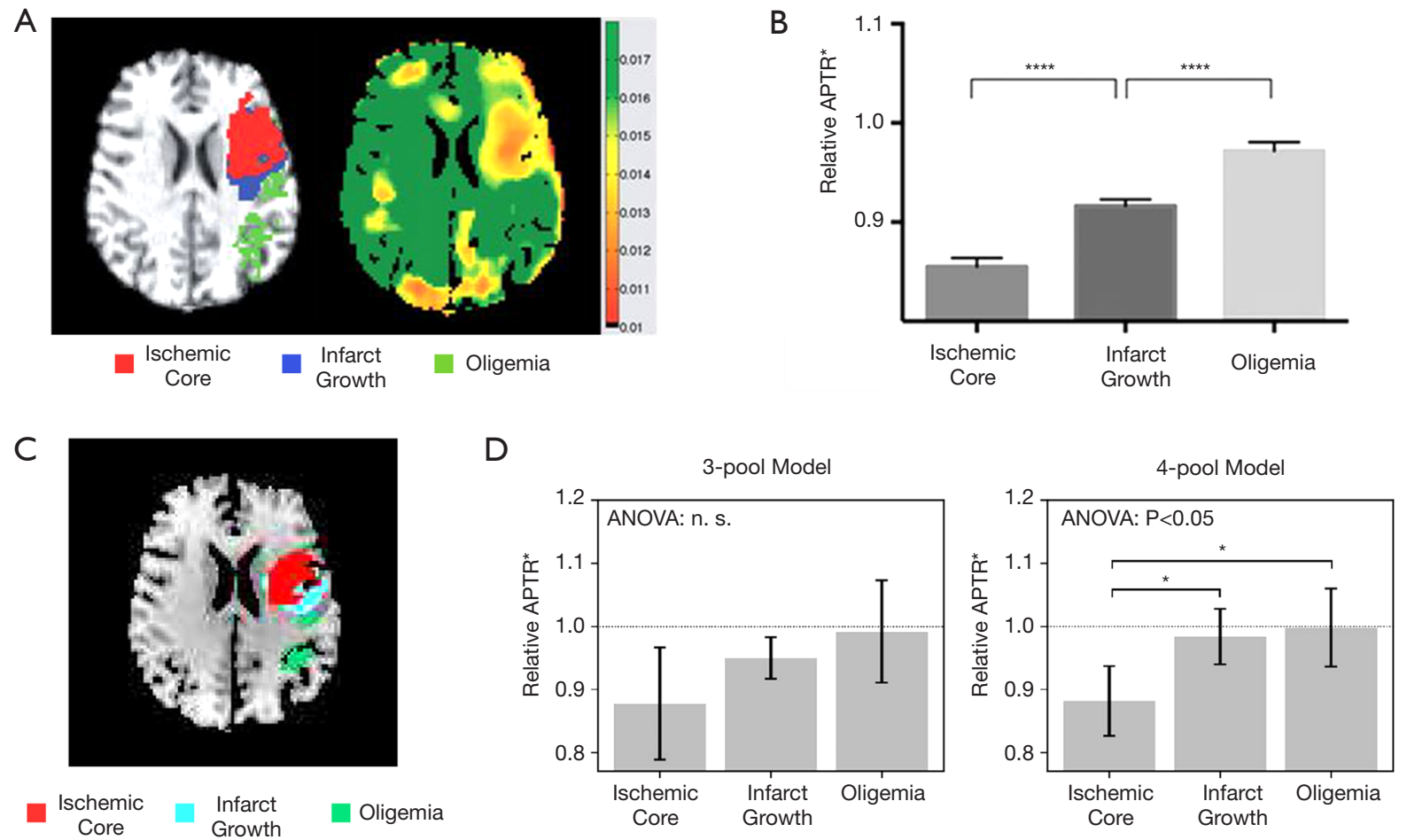

Figure 4 APT effect within different regions of the ischemic lesion. (A,B) Figures show the ROI defined, pH-weighted image, and quantified APT effects adapted from Harston et al. (33); (C,D) are the ROI defined, and quantified APT effects within the ROIs calculated using 3-pool and 4-pool Bloch McConnell model fitting, adapted from Msayib et al. (34). ${ }^{*} \mathrm{P}<0.05$; ${ }^{* * *} \mathrm{P}<0.0001$. APTR*, multi-pool BlochMcConnell model analysis. All images were reproduced with permission from the publishers.

investigated ROIs: ischemic core, final infarct growth and oligemia. Relative APT effect within the ischemic core was found to be significantly lower than within the penumbra which in turn was significantly lower than in the oligemia regions, as shown in Figure $4 B$. When compared to cerebral blood flow (CBF) and ADC, only APT was able to effectively distinguish between the penumbra and oligemia, although the comparisons with $\mathrm{ADC}$ and $\mathrm{CBF}$ were limited to just grey matter (33).

The study by Heo et al. (37) also observed positive results, where through the investigation of the spatial dynamics of the ischemic tissue, it was found that area of perfusion deficits was always larger than that of $\mathrm{pH}$ deficits-corresponding to the benign oligemia; $\mathrm{pH}$ deficits were equal to or larger than diffusion deficitscorresponding to the penumbra. The mean relative APT effect was also showed a decreasing trend from perfusion/ $\mathrm{pH}$ mismatch, $\mathrm{pH} /$ diffusion mismatch, to the infarct core (diffusion deficit only) (37).

Using data from the same patient cohort as the study by
Harston et al. (33), Msayib et al. (34) further investigated the differences between 3-pool and 4-pool Bloch McConnell model-based APT quantification. In Harston's study, a 3-pool Bloch McConnell model analysis was used (33). It was later verified in Msayib's study that although a 4-pool model would be more biophysically accurate, the 3-pool model was found to produce an intermediate infarct growth between the ischemic core and oligemia, proving to be more useful in the delineation of the penumbra (34). Despite this, there were no significant differences found between the three ROIs using the 3-pool model in conflict to Harston's study, although similar trends were observed (Figure 4D).

In short, Harston's (33) and Heo's (37) findings suggest that APT imaging may be able to improve the identification of ischemic penumbra in future clinical use, while Tietze's (36) and Msayib's (34) findings were inconclusive. The conflicting results between the studies may be attributed to several factors. Firstly, this may be a consequence of the small study sizes and the opportunity for single patients to create spurious results. Another possible source of 
contradiction is the use of different image acquisition schemes, quantification methods, and statistical approaches.

In the study by Tietze et al., the images obtained were three-dimensional (3D) images, while the other three studies assessed only single-slice images. It may be possible that the 3D CEST sequence used was not sufficiently sensitive to pick up on the variations within the different ischemic regions or that the quantification method used was not suitable for the sequence applied. Furthermore, absolute values were used for group analysis in the study which may not account for the different stroke severity and other variabilities. As previously pointed out, relative values are more suitable for groupwise comparisons (49).

Similarly, the discrepancies between the studies by Harston and Msayib may be attributed to the different fitting limits used during the Bloch McConnell model fitting and different statistical approaches taken. Although the model-based method is useful for quantifying the pure APT effect uncontaminated by other confounding effects, the model fitting process is typically dependent on the initial values and fitting boundaries, as well as image quality, thus results are more variable across different studies, particularly with CEST systems that require a large number of pools or fitting parameters.

Overall, the results of the four studies suggest it might be possible for APT imaging to delineate the three injury zones, but larger validation studies are needed to determine the optimal experimental parameters and the suitable APT quantification method.

\section{Limitations}

The main limitation faced by the clinical studies is the recruitment of patients and potential bias this can introduce. This is especially challenging in the hyperacute setting when early treatment is time-critical, and patients can find it difficult to tolerate research MRI scans. Follow-up imaging can also be hard to acquire since 1 in 8 strokes are fatal within the first 30 days and almost two-thirds of stroke result in disability (50). Consequently, the recruitment of patients for follow-up scans to confirm APT findings is commonly incomplete, or leads to inconsistent final infarct definitions being used (7).

Studies that aim to investigate APT effect across different stroke stages such as the studies by Song et al. (40), Yu et al. (30), and Momosaka et al. (43) face further challenges as the number of patients across different stages recruited should be sufficiently large to ensure repeatability and to minimize biases arising from a few dominant results. Although Lee et al. (41) demonstrated good promise in the reliability of APT imaging across different sessions, multicenter investigations with larger patient sample size are still needed to prove the repeatability of APT MRI in different clinical settings.

Furthermore, many of the studies reported to exclude some patient data for analysis due to severe motion artifacts, further reducing usable patient data $(29,30,33,40,42,43)$. Motion is an issue in MRI of stroke as patients may have difficulty to remain relatively still in the confined area for tens of minutes compared to CT imaging that is only a few minutes long. Most studies have made efforts to minimize patient motion or re-acquire corrupt acquisitions. Despite this, certain patient data still had to be excluded due to excessive motion.

Referring to https://cdn.amegroups.cn/static/public/ qims-20-1339-1.pdf, all the included studies were conducted using $3 \mathrm{~T}$ field strength scanners. Most MR scanners in the emergency room are currently $1.5 \mathrm{~T}$. As the APT effect would be smaller at lower field strengths due to smaller $T_{1}$ relaxation time $(51,52)$, it remains unclear if APT imaging of ischemic stroke can be translated to lower field strengths due to lower signal-to-noise ratio (SNR) (53). A recent study has shown that CEST imaging could work at $1.5 \mathrm{~T}$ for brain tumor imaging (54). However, the same has not been tested for clinical stroke imaging. To promote wider clinical adoption, the investigation of the performance of APT ischemic stroke imaging at lower field strengths would be beneficial and could be a potential research topic for future studies.

Other limitations of the studies concern the lack of standardization between the studies on the experimental methods such as the imaging acquisition schemes. Apart from Tietze's (36), Park's (39) and Lee's (41) studies, the remaining clinical studies obtained only single-slice images for assessment. This leaves remaining parts of the stroke lesion unanalyzed, necessarily biasing the results. Ideally, volumetric imaging would be more clinically useful in the evaluation of the ischemic tissue, especially for the identification of the ischemic penumbra.

Although the first clinical study by Zhao's group aimed to propose a standardized RF saturation scheme for APT imaging of stroke and brain tumor patients, subsequent clinical studies used differing saturation schemes. While the early studies used continuous wave (CW) saturation, majority of the following studies all used variations of pulsed or pseudocontinuous sequences owing to the limitations 
in the amplifier duty cycle and the specific absorption rate (SAR) limit. Early APT studies also tended to utilize high saturation powers in order to suppress the NOE effects, but lower saturation powers were used in some of the later studies to cater to the slow exchange rate of amide protons $[\sim 28 \mathrm{~Hz}(22)]$ in order to increase the sensitivity to the observed APT effect (36).

The APT quantification methods of the studies were also heterogeneous, further complicating the comparison of results between the different centers. Of the 14 clinical studies, four were on the comparison of different quantification methods to determine the optimal method for stroke imaging $(26,34,36,37)$. In general, the studies found model-based quantitative methods to produce higher CNRs between stroke lesions and normal tissue and were thus deemed more optimal for stroke imaging. However, CNR may not necessarily be an accurate reflection of the actual pathophysiology (44) and is thus not a reliable metric for determining the optimal quantification method. Further, the computation times of some of the model-based quantification methods used in the studies were too long and are unlikely to be clinically feasible for acute stroke imaging, particularly the multi-pool Bloch McConnell model-based methods (55-57).

\section{Future recommendations}

For successful clinical translation, experimental results must be consistently reproducible across different MRI systems by various vendors. Consequently, a great degree of standardization on the image acquisition and post-processing methods should be set for the effective comparison of results from different centers as APT effect has been shown to be highly dependent on experimental methods as well as the parameters used (58-60). Nevertheless, as it is currently too early in the development of APT MRI for the standardization of these methods, a few recommendations and suggestions are presented for future studies.

In terms of the imaging scheme, volumetric imaging is crucial for clinical application and should thus be a point of focus in future studies. Volumetric imaging can be done through either multislice $(61,62)$ or 3D imaging (63-65). Ideally, the optimal image acquisition method should be fast to accommodate short scan times, but still reserving adequate SNR for accurate APT quantification. Although there are several issues in the implementation of $3 \mathrm{D}$ APT imaging, such as saturation spillover effects, $\mathrm{T}_{1}$ relaxation, frequency drifting, and long image acquisition times, many studies have proposed potential methods or options to address these issues.

Saturation spillover effects and $T_{1}$ relaxation can be minimized using relaxation-compensated APT quantification methods, e.g., apparent exchange-dependent relaxation (AREX) which compensates for spillover effects and scales for $T_{1}(66,67)$. Studies have also proposed imaging sequences to correct for frequency drift both in real time (68) and during post-processing (69). Additionally, to achieve clinically viable CEST imaging times, several imaging acceleration methods have been developed (21), including snapshot imaging $(64,65)$, compressed sensing $(70,71)$, and variably-accelerated sensitivity encoding (72). Other studies have also proposed optimized protocols that shorten acquisition time whilst preserving adequate SNR $(65,67,73)$.

Future investigations of ischemic stroke imaging using these imaging schemes or similar fast $3 \mathrm{D}$ imaging sequences would be required to fully assess APT effect within the ischemic volume. This would be particularly crucial for the identification of the penumbral volume to compare the APT deficit volume with the DWI and PWI deficit volumes, as was done previously in animal stroke models (27).

As for the quantification method, further investigations are still needed to determine the optimal quantification method for ischemic stroke imaging. In order to produce physiologically meaningful results, it is recommended that the methods should not only be evaluated in terms of contrast, but also their correlations with the pathophysiology (46). Further, it is also important to consider quantification methods with clinically feasible processing times. For now, it is suggested that future studies should report the most commonly used magnetization transfer ratio asymmetry, $\mathrm{MTR}_{\text {asym }}(3.5 \mathrm{ppm})$ results along with the findings of the study in order to ease the comparison of results of future publications and the existing literature.

Finally, to minimize exclusion of patient data due to motion, it is recommended that a new registration framework be designed. Thus far in the literature, several different registration tools have been used for registering CEST images, e.g., the built-in function "imregister" in MATLAB (Mathworks, Natick, MA, USA), medical imaging interaction toolkit (MITK), statistical parametric mapping (SPM), and MCFLIRT in the FMRIB Software Library. These software or functions improve the alignment of the CEST images and minimize the effects of motion. 
However, some of the registration tools utilize optimization methods that are prone to convergence to the local minima, resulting in inaccurate registration (74). Development of a new registration method or framework may help to improve the registration of motion-affected images and reduce the amount of data excluded due to the artifacts. Coupling this with the development of fast-volumetric imaging is one of the important milestones to translate APT MRI for clinical stroke imaging.

\section{Conclusions}

Since the first preclinical study in 2003, 14 small clinical studies have been published demonstrating the potential uses of APT MRI in ischemic stroke imaging, including the identification of the ischemic penumbra, predicting patient prognosis, and monitoring treatment in patients with ischemic stroke. Although APT imaging has shown some promise in these applications, further development and larger investigations are still needed to verify the clinical opportunity offered by the translation of APT imaging into clinical practice. This is because patient characteristics, image acquisition and post-processing methods are heterogenous, minimizing the conclusions that can be drawn from the existing evidences. It is recommended that future studies should report the results of $\mathrm{MTR}_{\text {asym }}$ (3.5 $\mathrm{ppm}$ ) alongside other findings to reduce this contribution to the heterogeneity observed and to enable comparison of results between studies and centers. Future investigations should also focus on the development of volumetric imaging for full ischemic tissue assessment to faciliate clinical translation.

\section{Acknowledgments}

Funding: This work was supported by National Cancer Council Malaysia (MAKNA) Cancer Research Award 2018 awarded to YKT; UTAR Research Fund [project number: IPSR/RMC/UTARRF/2018-C1/T04 to YKT, IPSR/ RMC/UTARRF/2020-C1/T02 to YKT]; and NVIDIA Corporation with the donation of a Quadro P6000 GPU to YKT.

\section{Footnote}

Conflicts of Interest: All authors have completed the ICMJE uniform disclosure form (available at http:// dx.doi.org/10.21037/qims-20-1339). YKT is supported by National Cancer Council Malaysia (MAKNA) Cancer Research Award 2018, UTAR Research Fund [project number: IPSR/RMC/UTARRF/2018-C1/T04, IPSR/ RMC/UTARRF/2020-C1/T02], and NVIDIA Corporation with the donation of a Quadro P6000 GPU. GH is part time employed by and has stock options with Brainomix Ltd. The other authors have no conflicts of interest to declare.

Ethical Statement: The authors are accountable for all aspects of the work in ensuring that questions related to the accuracy or integrity of any part of the work are appropriately investigated and resolved.

Open Access Statement: This is an Open Access article distributed in accordance with the Creative Commons Attribution-NonCommercial-NoDerivs 4.0 International License (CC BY-NC-ND 4.0), which permits the noncommercial replication and distribution of the article with the strict proviso that no changes or edits are made and the original work is properly cited (including links to both the formal publication through the relevant DOI and the license). See: https://creativecommons.org/licenses/by-nc-nd/4.0/.

\section{References}

1. Stroke Statistics [Internet]. The Internet Stroke Center. [cited 2020 Jul 30]. Available online: http://www. strokecenter.org/patients/about-stroke/stroke-statistics/

2. Ischemic Strokes (Clots) [Internet]. American Heart Association. [cited $2020 \mathrm{Jul}$ 30]. Available online: https:// www.stroke.org/en/about-stroke/types-of-stroke/ ischemic-stroke-clots

3. Mozaffarian D, Benjamin EJ, Go AS, Arnett DK, Blaha MJ, Cushman M, Das SR, Ferranti S De, Després JP, Fullerton HJ, Howard VJ, Huffman MD, Isasi CR, Jiménez MC, Judd SE, Kissela BM, Lichtman JH, Lisabeth LD, Liu S, MacKey RH, Magid DJ, McGuire DK, Mohler ER, Moy CS, Muntner P, Mussolino ME, Nasir K, Neumar RW, Nichol G, Palaniappan L, Pandey DK, Reeves MJ, Rodriguez CJ, Rosamond W, Sorlie PD, Stein J, Towfighi A, Turan TN, Virani SS, Woo D, Yeh RW, Turner MB. Heart disease and stroke statistics-2016 update a report from the American Heart Association. Circulation 2016;133:e38-360.

4. Catanese L, Tarsia J, Fisher M. Acute Ischemic Stroke Therapy Overview. Circ Res 2017;120:541-58.

5. Campbell BCV, De Silva DA, Macleod MR, Coutts SB, 
Schwamm LH, Davis SM, Donnan GA. Ischaemic stroke. Nat Rev Dis Prim 2019;5:70.

6. Wardlaw JM. Surrogate outcomes a cautionary note. Stroke 2009;40:1029-31.

7. Harston GWJ, Rane N, Shaya G, Thandeswaran S, Cellerini M, Sheerin F, Kennedy J. Imaging biomarkers in acute ischemic stroke trials: a systematic review. AJNR Am J Neuroradiol 2015;36:839-43.

8. Astrup J, Siesjö BK, Symon L. Thresholds in cerebral ischemia - the ischemic penumbra. Stroke 1981;12:723-5.

9. Huisa BN, Neil WP, Schrader R, Maya M, Pereira B, Bruce NT, Lyden PD. Clinical use of computed tomographic perfusion for the diagnosis and prediction of lesion growth in acute ischemic stroke. J Stroke Cerebrovasc Dis 2014;23:114-22.

10. Lin MP, Liebeskind DS. Imaging of Ischemic Stroke. Continuum (Minneap Minn) 2016;22:1399-423.

11. Demeestere J, Wouters A, Christensen S, Lemmens R, Lansberg MG. Review of Perfusion Imaging in Acute Ischemic Stroke. Stroke 2020;51:1017-24.

12. Leigh R, Knutsson L, Zhou J, van Zijl PCM. Imaging the physiological evolution of the ischemic penumbra in acute ischemic stroke. J Cereb Blood Flow Metab 2018;38:1500-16.

13. Guadagno JV, Warburton EA, Jones PS, Fryer TD, Day DJ, Gillard JH, Carpenter TA, Aigbirhio FI, Price CJ, Baron JC. The diffusion-weighted lesion in acute stroke: Heterogeneous patterns of flow/metabolism uncoupling as assessed by quantitative positron emission tomography. Cerebrovasc Dis 2005;19:239-46.

14. Martins N, Aires A, Mendez B, Boned S, Rubiera M, Tomasello A, Coscojuela P, Hernandez D, Muchada M, Rodríguez-Luna D, Rodríguez N, Juega JM, Pagola J, Molina CA, Ribó M. Ghost Infarct Core and Admission Computed Tomography Perfusion: Redefining the Role of Neuroimaging in Acute Ischemic Stroke. Interv Neurol 2018;7:513-21.

15. Hossmann KA. Viability thresholds and the penumbra of focal ischemia. Ann Neurol 1994;36:557-65.

16. Ward KM, Balaban RS. Determination of $\mathrm{pH}$ using water protons and Chemical Exchange Dependent Saturation Transfer (CEST). Magn Reson Med 2000;44:799-802.

17. Ward KM, Aletras AH, Balaban RS. A New Class of Contrast Agents for MRI Based on Proton Chemical Exchange Dependent Saturation Transfer (CEST). J Magn Reson 2000;143:79-87.

18. van Zijl PC, Yadav NN. Chemical exchange saturation transfer (CEST): What is in a name and what isn't? Magn Reson Med 2011;65:927-48.

19. Jokivarsi KT, Gröhn HI, Gröhn OH, Kauppinen RA. Proton transfer ratio, lactate, and intracellular $\mathrm{pH}$ in acute cerebral ischemia. Magn Reson Med 2007;57:647-53.

20. Zhou J, van Zijl PCM. Defining an Acidosis-Based Ischemic Penumbra from pH-Weighted MRI. Transl Stroke Res 2011;3:76-83.

21. Zhou J, Heo HY, Knutsson L, van Zijl PCM, Jiang S. APT-weighted MRI: Techniques, current neuro applications, and challenging issues. J Magn Reson Imaging 2019;50:347-64.

22. Zhou J, Payen JF, Wilson DA, Traystman RJ, Van Zijl PCM. Using the amide proton signals of intracellular proteins and peptides to detect $\mathrm{pH}$ effects in MRI. Nat Med 2003;9:1085-90.

23. Erecińska M, Silver IA. ATP and brain function. J Cereb Blood Flow Metab 1989;9:2-19.

24. Hossmann KA. Pathophysiological basis of translational stroke research. Folia Neuropathol 2009;47:213-27.

25. Sun PZ, Zhou J, Sun W, Huang J, Van Zijl PCM. Detection of the ischemic penumbra using $\mathrm{pH}$-weighted MRI. J Cereb Blood Flow Metab 2007;27:1129-36.

26. Tee YK, Harston GWJ, Blockley N, Okell TW, Levman J, Sheerin F, Cellerini M, Jezzard P, Kennedy J, Payne SJ, Chappell MA. Comparing different analysis methods for quantifying the MRI amide proton transfer (APT) effect in hyperacute stroke patients. NMR Biomed 2014;27:1019-29.

27. Guo Y, Zhou IY, Chan ST, Wang Y, Mandeville ET, Igarashi T, Lo EH, Ji X, Sun PZ. pH-sensitive MRI demarcates graded tissue acidification during acute stroke - $\mathrm{pH}$ specificity enhancement with magnetization transfer and relaxation-normalized amide proton transfer (APT) MRI. Neuroimage 2016;141:242-9.

28. Sun PZ. Fast correction of B0 field inhomogeneity for $\mathrm{pH}$-specific magnetization transfer and relaxation normalized amide proton transfer imaging of acute ischemic stroke without Z-spectrum. Magn Reson Med 2020;83:1688-97.

29. Lin G, Zhuang C, Shen Z, Xiao G, Chen Y, Shen Y, Zong $\mathrm{X}, \mathrm{Wu}$ R. APT weighted MRI as an effective imaging protocol to predict clinical outcome after acute ischemic stroke. Front Neurol 2018;9:901.

30. Yu L, Chen Y, Chen M, Luo X, Jiang S, Zhang Y, Chen H, Gong T, Zhou J, Li C. Amide proton transfer MRI signal as a surrogate biomarker of ischemic stroke recovery in patients with supportive treatment. Front Neurol 
2019;10:104.

31. Liberati A, Altman DG, Tetzlaff J, Mulrow C, Gøtzsche PC, Ioannidis JPA, Clarke M, Devereaux PJ, Kleijnen J, Moher D. The PRISMA statement for reporting systematic reviews and meta-analyses of studies that evaluate health care interventions: explanation and elaboration. J Clin Epidemiol 2009;62:e1-34.

32. Bernhardt J, Hayward KS, Kwakkel G, Ward NS, Wolf SL, Borschmann K, Krakauer JW, Boyd LA, Carmichael ST, Corbett D, Cramer SC. Agreed Definitions and a Shared Vision for New Standards in Stroke Recovery Research: The Stroke Recovery and Rehabilitation Roundtable Taskforce. Neurorehabil Neural Repair 2017;31:793-9.

33. Harston GWJ, Tee YK, Blockley N, Okell TW, Thandeswaran S, Shaya G, Sheerin F, Cellerini M, Payne S, Jezzard P, Chappell M, Kennedy J. Identifying the ischaemic penumbra using $\mathrm{pH}$-weighted magnetic resonance imaging. Brain 2015;138:36-42.

34. Msayib Y, Harston GWJ, Tee YK, Sheerin F, Blockley NP, Okell TW, Jezzard P, Kennedy J, Chappell MA. Quantitative CEST imaging of amide proton transfer in acute ischaemic stroke. NeuroImage Clin 2019;23:101833.

35. Msayib Y, Harston GWJ, Sheerin F, Blockley NP, Okell TW, Jezzard P, Kennedy J, Chappell MA. Partial volume correction for quantitative CEST imaging of acute ischemic stroke. Magn Reson Med 2019;82:1920-8.

36. Tietze A, Blicher J, Mikkelsen IK, Østergaard L, Strother MK, Smith SA, Donahue MJ. Assessment of ischemic penumbra in patients with hyperacute stroke using amide proton transfer (APT) chemical exchange saturation transfer (CEST) MRI. NMR Biomed 2014;27:163-74.

37. Heo HY, Zhang Y, Burton TM, Jiang S, Zhao Y, van Zijl PCM, Leigh R, Zhou J. Improving the detection sensitivity of $\mathrm{pH}$-weighted amide proton transfer MRI in acute stroke patients using extrapolated semisolid magnetization transfer reference signals. Magn Reson Med 2017;78:871-80.

38. Zhao X, Wen Z, Huang F, Lu S, Wang X, Hu S, Zu D, Zhou J. Saturation power dependence of amide proton transfer image contrasts in human brain tumors and strokes at 3 T. Magn Reson Med 2011;66:1033-41.

39. Park JE, Kim HS, Jung SC, Keupp J, Jeong H-K, Kim SJ. Depiction of Acute Stroke Using 3-Tesla Clinical Amide Proton Transfer Imaging: Saturation Time Optimization Using an in vivo Rat Stroke Model, and a Preliminary Study in Human. Investig Magn Reson Imaging 2017;21:65-70.
40. Song G, Li C, Luo X, Zhao X, Zhang S, Zhang Y, Jiang S, Wang X, Chen Y, Chen H, Gong T, Zhou J, Chen M. Evolution of cerebral ischemia assessed by amide proton transfer-weighted MRI. Front Neurol 2017;8:67.

41. Lee JB, Park JE, Jung SC, Jo Y, Kim D, Kim HS, Choi CG, Kim SJ, Kang DW. Repeatability of amide proton transfer-weighted signals in the brain according to clinical condition and anatomical location. Eur Radiol 2020;30:346-56.

42. Wang Z, Shaghaghi M, Zhang S, Zhang G, Zhou Y, Wu D, Zhang Z, Zhu W, Cai K. Novel proton exchange rate MRI presents unique contrast in brains of ischemic stroke patients. J Neurosci Methods 2020;346:108926.

43. Momosaka D, Togao O, Kikuchi K, Kikuchi Y, Wakisaka Y, Hiwatashi A. Correlations of amide proton transferweighted MRI of cerebral infarction with clinicoradiological findings. PLoS One 2020;15:e0237358.

44. Wen Z, Hu S, Huang F, Wang X, Guo L, Quan X, Wang $\mathrm{S}$, Zhou J. MR imaging of high-grade brain tumors using endogenous protein and peptide-based contrast. Neuroimage 2010;51:616-22.

45. Li C, Peng S, Wang R, Chen H, Su W, Zhao X, Zhou J, Chen M. Chemical exchange saturation transfer MR imaging of Parkinson's disease at 3 Tesla. Eur Radiol 2014;24:2631-9.

46. Foo LS, Larkin JR, Sutherland BA, Ray KJ, Yap WS, Hum YC, Lai KW, Manan HA, Sibson NR, Tee YK. Study of common quantification methods of amide proton transfer magnetic resonance imaging for ischemic stroke detection. Magn Reson Med 2021;85:2188-200.

47. Zhou IY, Lu D, Ji Y, Wu L, Wang E, Cheung JS, Zhang XA, Sun PZ. Determination of multipool contributions to endogenous amide proton transfer effects in global ischemia with high spectral resolution in vivo chemical exchange saturation transfer MRI. Magn Reson Med 2019;81:645-52.

48. Wu Y, Zhou IY, Lu D, Manderville E, Lo EH, Zheng H, Sun PZ. pH-sensitive amide proton transfer effect dominates the magnetization transfer asymmetry contrast during acute ischemia-quantification of multipool contribution to in vivo CEST MRI. Magn Reson Med 2018;79:1602-8.

49. Tee YK, Abidin B, Khrapitchev A, Sutherland BA, Larkin J, Ray K, Harston GWJ, Buchan AM, Kennedy J, Sibson NR, Chappell MA. CEST and NOE signals in ischemic stroke at 9.4T evaluated using a Lorentzian multi-pool analysis: a drop, an increase or no change? In: Proc Int Soc Magn Reson Med 2017. p. 5-6. 
50. State of the Nation Stroke Statistics [Internet]. The Stroke Association. 2018 [cited 2019 Jul 3]. Available online: https://www.stroke.org.uk/system/files/sotn_2018.pdf

51. Woessner DE, Zhang S, Merritt ME, Sherry AD. Numerical solution of the Bloch equations provides insights into the optimum design of PARACEST agents for MRI. Magn Reson Med 2005;53:790-9.

52. Tee YK, Donahue MJ, Harston GWJ, Payne SJ, Chappell MA. Quantification of amide proton transfer effect preAnd post-gadolinium contrast agent administration. J Magn Reson Imaging 2014;40:832-8.

53. Foo LS, Yap WS, Hum YC, Manan HA, Tee YK. Analysis of model-based and model-free CEST effect quantification methods for different medical applications. J Magn Reson 2020;310:106648.

54. Chan RW, Chen H, Myrehaug S, Atenafu EG, Stanisz GJ, Stewart J, Maralani PJ, Chan AKM, Daghighi S, Ruschin M, Das S, Perry J, Czarnota GJ, Sahgal A, Lau AZ. Quantitative CEST and MT at 1.5T for monitoring treatment response in glioblastoma: early and late tumor progression during chemoradiation. J Neurooncol 2021;151:267-78.

55. Tee YK, Khrapitchev AA, Sibson NR, Payne SJ, Chappell MA. Evaluating the use of a continuous approximation for model-based quantification of pulsed chemical exchange saturation transfer (CEST). J Magn Reson 2012;222:88-95.

56. Chappell MA, Donahue MJ, Tee YK, Khrapitchev AA, Sibson NR, Jezzard P, Payne SJ. Quantitative Bayesian model-based analysis of amide proton transfer MRI. Magn Reson Med 2013;70:556-67.

57. Foo LS, Yap WS, Tee YK. Determination of computationally efficient multi-pool model fitting approach for pulsed chemical exchange saturation transfer MRI. In: ACM International Conference Proceeding Series Beijing: ACM; 2019. p. 32-9.

58. Schmidt H, Schwenzer NF, Gatidis S, Küstner T, Nikolaou K, Schick F, Martirosian P. Systematic Evaluation of Amide Proton Chemical Exchange Saturation Transfer at 3 T: Effects of Protein Concentration, $\mathrm{pH}$, and Acquisition Parameters. Invest Radiol 2016;51:635-46.

59. Heo HY, Lee DH, Zhang Y, Zhao X, Jiang S, Chen M, Zhou J. Insight into the quantitative metrics of chemical exchange saturation transfer (CEST) imaging. Magn Reson Med 2017;77:1853-65.

60. Heo HY, Zhang Y, Jiang S, Zhou J. Influences of experimental parameters on chemical exchange saturation transfer (CEST) metrics of brain tumors using animal models at 4.7T. Magn Reson Med 2019;81:316-30.

61. Randtke EA, Granados JC, Howison CM, Pagel MD, Cárdenas-Rodríguez J. Multislice CEST MRI improves the spatial assessment of tumor pH. Magn Reson Med 2017;78:97-106.

62. Cai J, Wu J, Guo C, Cai S, Cai C. Ultrafast multi-slice chemical exchange saturation transfer imaging scheme based on segmented spatiotemporal encoding. Magn Reson Imaging 2019;60:122-9.

63. Zhu H, Jones CK, Van Zijl PCM, Barker PB, Zhou J. Fast 3D chemical exchange saturation transfer (CEST) imaging of the human brain. Magn Reson Med 2010;64:638-44.

64. Deshmane A, Zaiss M, Lindig T, Herz K, Schuppert M, Gandhi C, Bender B, Ernemann U, Scheffler K. 3D gradient echo snapshot CEST MRI with low power saturation for human studies at 3T. Magn Reson Med 2019;81:2412-23.

65. Mueller S, Stirnberg R, Akbey S, Ehses P, Scheffler K, Stöcker T, Zaiss M. Whole brain snapshot CEST at $3 \mathrm{~T}$ using 3D-EPI: Aiming for speed, volume, and homogeneity. Magn Reson Med 2020;84:2469-83.

66. Zaiss M, Xu J, Goerke S, Khan IS, Singer RJ, Gore JC, Gochberg DF, Bachert P. Inverse Z-spectrum analysis for spillover-, MT-, and T1-corrected steady-state pulsed CEST-MRI - application to pH-weighted MRI of acute stroke. NMR Biomed 2014;27:240-52.

67. Goerke S, Breitling J, Korzowski A, Paech D, Zaiss M, Schlemmer H-P, Ladd ME, Bachert P. Clinical routine acquisition protocol for $3 \mathrm{D}$ relaxation-compensated $\mathrm{APT}$ and rNOE CEST-MRI of the human brain at 3T. Magn Reson Med 2021;86:393-404.

68. Liu R, Zhang H, Niu W, Lai C, Ding Q, Chen W, Liang S, Zhou J, Wu D, Zhang Y. Improved chemical exchange saturation transfer imaging with real-time frequency drift correction. Magn Reson Med 2019;81:2915-23.

69. Windschuh J, Zaiss M, Ehses P, Lee JS, Jerschow A, Regatte RR. Assessment of frequency drift on CEST MRI and dynamic correction: application to gagCEST at $7 \mathrm{~T}$. Magn Reson Med 2019;81:573-82.

70. Heo HY, Zhang Y, Lee DH, Jiang S, Zhao X, Zhou J. Accelerating chemical exchange saturation transfer (CEST) MRI by combining compressed sensing and sensitivity encoding techniques. Magn Reson Med 2017;77:779-86.

71. Heo HY, Xu X, Jiang S, Zhao Y, Keupp J, Redmond KJ, Laterra J, van Zijl PCM, Zhou J. Prospective acceleration of parallel RF transmission-based 3D chemical exchange saturation transfer imaging with compressed sensing. 
Magn Reson Med 2019;82:1812-21.

72. Zhang Y, Heo HY, Lee DH, Jiang S, Zhao X, Bottomley PA, Zhou J. Chemical exchange saturation transfer (CEST) imaging with fast variably-accelerated sensitivity encoding (vSENSE). Magn Reson Med 2017;77:2225-38.

73. Tee YK, Khrapitchev AA, Sibson NR, Payne SJ, Chappell MA. Optimal sampling schedule for chemical exchange

Cite this article as: Foo LS, Harston G, Mehndiratta A, Yap WS, Hum YC, Lai KW, Mohamed Mukari SA, Mohd Zaki F, Tee YK. Clinical translation of amide proton transfer (APT) MRI for ischemic stroke: a systematic review (2003-2020). Quant Imaging Med Surg 2021;11(8):3797-3811. doi: 10.21037/ qims-20-1339 saturation transfer. Magn Reson Med 2013;70:1251-62 .

74. Liang Y, Bie C, Chen B, Hou Y, Song X. Motion correction in CEST MRI series exploiting Adaptive Stochastic Gradient Descent (ASGD) - Based optimization algorithm. In: 2019 International Conference on Medical Imaging Physics and Engineering, ICMIPE 20192019. 